\title{
Premature Stroke Secondary to Severe Hypertension Results from Liddle Syndrome Caused by a Novel SCNN1B Mutation
}

\author{
Peng Fan $^{\mathrm{a}}$ Di Zhang ${ }^{\mathrm{b}}$ Xiao-Cheng Pan $^{\mathrm{c}}$ Kun-Qi Yang ${ }^{\mathrm{a}}$

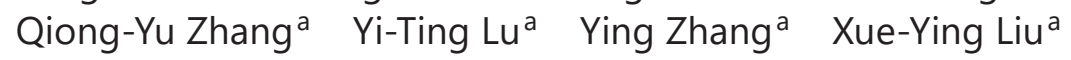 \\ Wen-Jun Ma ${ }^{a}$ Hui-Min Zhang ${ }^{a}$ Lei Song ${ }^{a}$ Jun Cai ${ }^{a}$ \\ Ya-Xin Liu ${ }^{\mathrm{b}}$ Xian-Liang Zhou ${ }^{\mathrm{a}}$ \\ aDepartment of Cardiology, Fuwai Hospital, National Center for Cardiovascular Diseases, \\ Chinese Academy of Medical Sciences and Peking Union Medical College, Beijing, China; \\ ${ }^{b}$ Emergency and Critical Care Center, Fuwai Hospital, National Center for Cardiovascular \\ Diseases, Chinese Academy of Medical Sciences and Peking Union Medical College, \\ Beijing, China; ' 'Graduate School, Peking Union Medical College and Chinese Academy of \\ Medical Sciences, Beijing, China
}

\section{Keywords}

Liddle syndrome $\cdot$ SCNN1B mutation · Hypertension · Stroke

\begin{abstract}
Introduction: Liddle syndrome (LS), an autosomal dominant and inherited monogenic hypertension syndrome caused by pathogenic mutations in the epithelial sodium channel (ENaC) genes SCNN1A, SCNN1B, and SCNN1G. Objective: This study was designed to identify a novel SCNN1B missense mutation in a Chinese family with a history of stroke, and to confirm that the identified mutation is responsible for LS in this family. Methods: DNA samples were collected from the proband and 11 additional relatives. Next-generation sequencing was performed in the proband to find candidate variants. In order to exclude genetic polymorphism, the candidate variant in SCNN1B was verified in other family members, 100 hypertensives, and 100 healthy controls by Sanger sequencing. Results: Genetic testing revealed a novel and rare heterozygous variant in SCNN1B in the proband. This variant resulted in a substitution of threonine instead of proline at codon 617 , altering the PY motif of $\beta$-ENaC. The identified mutation was only verified in 5 relatives. In silico analyses indicated that this variant was highly pathogenic. In this family, phenotypic heterogeneity was present among 6 LS patients. Tailored medicine with amiloride was effective in controlling hypertension and improving the
\end{abstract}

Peng Fan and Di Zhang contributed equally to this work.

Ya-Xin Liu

Emergency and Critical Care Center, Fuwai Hospital, National Center for Cardiovascular Diseases, Chinese Academy of Medical Sciences and Peking Union Medical College, No. 167, Beilishi Rd., Beijing 100037 (China) yaxinliu1978@ hotmail.com
Xian-Liang Zhou

Department of Cardiology, Fuwai Hospital National Center for Cardiovascular Diseases Chinese Academy of Medical Sciences and Peking Union Medical College

No. 167, Beilishi Rd., Beijing 100037 (China) zhouxianliang0326@ hotmail.com 


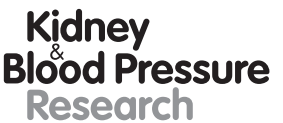



Fan et al.: LS Caused by a Novel SCNN1B Mutation

serum potassium concentration in patients with LS. Conclusions: We identified a novel SCNN1B mutation (c.1849C >A) in a family affected by LS. Patients with LS, especially those with severe hypertension, should be alert for the occurrence of premature stroke. Timely diagnosis using genetic testing and tailored treatment with amiloride can help LS patients to avoid severe complications.

\section{Introduction}

Hypertension affects approximately 1 billion people worldwide [1] and it is the most prevalent risk factor for cardiovascular diseases and related disabilities [2]. A minority of patients with hypertension have an inherited disorder, and monogenic hypertension syndromes are specific types of secondary hypertension [3, 4]. Among these diseases, Liddle syndrome (LS) is relatively rare, and the prevalence of LS across the general hypertensive population is unknown. Previous studies revealed that the prevalence of LS was $0.91 \sim 1.52 \%$ after exclusion of the most common secondary forms of hypertension $[5,6]$.

LS, first reported by Liddle [7] in 1963, is a rare autosomal dominant monogenic form of hypertension [8]. Affected patients typically present with early-onset hypertension, hypokalemia, and suppressed plasma renin activity, as well as hypoaldosteronism and metabolic alkalosis. A potassium-sparing diuretic (amiloride or triamterene) can improve the disease [9]. LS is caused by pathogenic mutations in the SCNN1A, SCNN1B, and SCNN1G genes, which encode the $\alpha, \beta$, and $\gamma$ subunits of the epithelial sodium channel (ENaC) [10], respectively. Genetic sequencing is a rapid and accurate tool recommended for patients in whom LS is suspected. The absence of genetic sequencing and genotype-phenotype heterogeneity mean that some patients with LS are misdiagnosed [9]. The severe complications caused by LS, including heart failure, cerebral hemorrhage, and premature death at an early age, are common [11].

In this study, we identified a Chinese family with LS, caused by a novel missense mutation (c.1849C $>$ A) in $S C N N 1 B$, leading to the replacement of proline 617 with threonine. The genotype-phenotype analysis revealed heterogeneity among the pedigree members, especially in terms of hypokalemia, and a low-renin concentration. Genetic diagnosis and tailored medicine can prevent serious complications resulting from poor blood pressure and potassium level control.

\section{Materials and Methods}

\section{Subjects}

The proband (III-8), a 22-year-old male with early-onset and refractory hypertension, hypokalemia, suppressed plasma renin (PRC) and plasma aldosterone concentrations (PAC), and a history of stroke, was admitted to the Department of Hypertension at Fuwai Hospital. The other 11 family members (Fig. 1) also underwent biochemical and genetic analyses. One hundred hypertensives and 100 healthy controls were enrolled into this study.

\section{Clinical and Biochemical Features}

Computed tomography of the kidneys, adrenal glands, and renal arteries and echocardiography were performed on the proband. PRC and PAC were measured in 8 subjects (Table 1) after they had remained in an upright position for $2 \mathrm{~h}$. PRC and PAC were measured by 


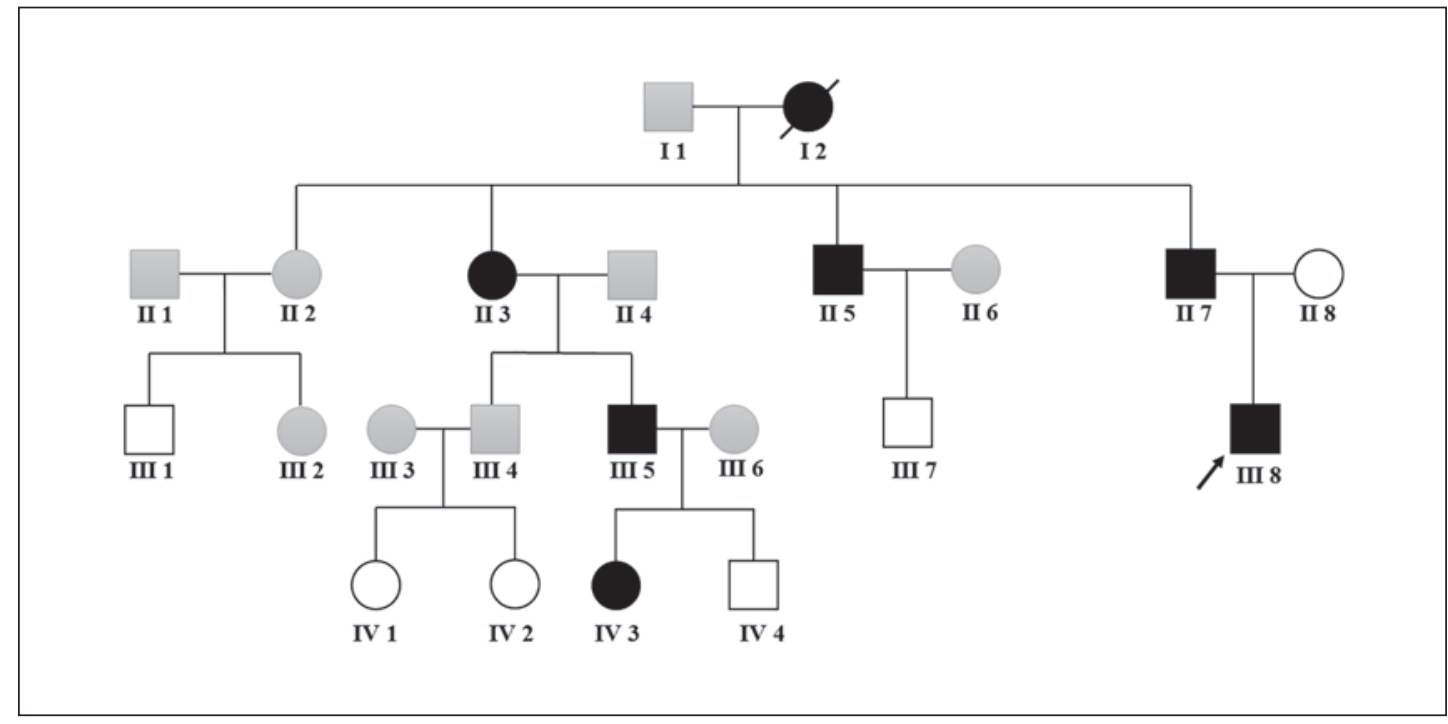

Fig. 1. Patients with LS carrying the mutation are indicated by black symbols. Six family members without the mutation are indicated by white symbols. Others without genetic sequencing are indicated by gray symbols. The arrow marks the proband, and the deceased family member is indicated by a diagonal line.

chemiluminescence immunoassay using a LIAISON Direct Renin kit (DiaSorin S.p.A, Vercelli, Italy) and a LIAISON Aldosterone kit (DiaSorin Inc., Stillwater, MN, USA), respectively. Other biochemical measurements were made using standard methods.

\section{Genetic Screening}

Genomic DNA was extracted from the peripheral blood leucocytes of all subjects using a QIA Amp DNA Blood Mini kit (QIAGEN, Hilden, Germany). Next-generation sequencing was performed on the proband using a panel (online suppl. Table 1; for online suppl. material, see www.karger.com/doi/10.1159/000507580) containing 101 genes associated with monogenic hypertension. The candidate $S C N N 1 B$ variant was verified by Sanger sequencing. In order to exclude polymorphism, the identified mutation also detected in the other 11 family members, 100 hypertensives, and 100 healthy controls. Polymerase chain reaction (PCR) was used to amplify exon 13 of SCNN1B using a gene-specific primer pair designed by Yang et al. [11] (forward primer: 5'-CAAGAATCACCTCCCAGGAAGC-3', reverse primer: 5'-GTGATTCTACAGAGCCCTTGGC-3'). All PCR products were sequenced using an ABI Prism 377 DNA sequencer (Applied Biosystems, Foster City, CA, USA).

\section{Results}

\section{Clinical Features}

The proband had suffered a sudden cerebral hemorrhage secondary to severe hypertension (220/140 $\mathrm{mm} \mathrm{Hg}) 3$ months prior to admission. It was the first time he realized he suffered hypertension and hypokalemia. Although he had had symptoms of headache and dizziness frequently over the past several years, these disorders did not draw his attention for further physical examination. His blood pressure was not efficiently controlled after taking nifedipine, irbesartan hydrochlorothiazide, and fosinopril. His serum potassium levels varied from 2.70 to $3.24 \mathrm{mmol} / \mathrm{L}$ over 3 months. Therefore, he was admitted to the department 
Table 1. Clinical and biochemical characteristics of 12 subjects in this family

\begin{tabular}{|c|c|c|c|c|c|c|c|c|c|}
\hline \multirow[t]{2}{*}{ Subject } & \multirow{2}{*}{$\begin{array}{l}\text { Age, } \\
\text { years }\end{array}$} & \multirow[t]{2}{*}{ Gender } & \multirow{2}{*}{$\begin{array}{l}\text { Onset age of } \\
\text { hypertension, } \\
\text { years }\end{array}$} & \multirow{2}{*}{$\begin{array}{l}\text { Maximum BP, } \\
\mathrm{mm} \mathrm{Hg}\end{array}$} & \multirow{2}{*}{$\begin{array}{l}\text { Serum } \mathrm{K}^{+}, \\
\mathrm{mmol} / \mathrm{L}\end{array}$} & \multirow{2}{*}{$\begin{array}{l}\text { Serum PRC }{ }^{2} \text {, } \\
\mu \mathrm{IU} / \mathrm{mL}\end{array}$} & \multirow{2}{*}{$\begin{array}{l}\text { Serum } P A^{2} \text {, } \\
n g / d L\end{array}$} & \multicolumn{2}{|c|}{ Follow-up } \\
\hline & & & & & & & & $\begin{array}{l}\mathrm{BP}, \\
\mathrm{mm} \mathrm{Hg}\end{array}$ & $\begin{array}{l}\text { serum } \mathrm{K}^{+}, \\
\mathrm{mmol} / \mathrm{L}\end{array}$ \\
\hline \multicolumn{10}{|l|}{ Affected $^{1}$} \\
\hline II-3 & 58 & $\mathrm{~F}$ & 20 & $200 / 150$ & 3.01 & 24.70 & 2.90 & $130 / 80$ & 4.43 \\
\hline II-5 & 50 & M & 15 & $220 / 120$ & 2.80 & 1.10 & 1.70 & $133 / 87$ & 4.38 \\
\hline II-7 & 49 & M & 20 & $180 / 120$ & 3.41 & 20.30 & 1.90 & $126 / 80$ & 4.73 \\
\hline III-5 & 34 & M & 20 & $190 / 130$ & 3.12 & 3.20 & 2.60 & $130 / 80$ & 4.66 \\
\hline III-8 & 22 & M & 22 & $220 / 140$ & 2.54 & 69.30 & 1.50 & $125 / 80$ & 3.79 \\
\hline IV-3 & 8 & $\mathrm{~F}$ & 8 & $150 / 100$ & 4.15 & 4.20 & 2.70 & $115 / 70$ & 4.76 \\
\hline \multicolumn{10}{|c|}{ Unaffected } \\
\hline II-8 & 49 & $\mathrm{~F}$ & - & $134 / 80$ & 3.96 & 20.60 & 6.60 & - & - \\
\hline III-1 & 36 & M & - & $145 / 95$ & 4.33 & - & - & - & - \\
\hline III-7 & 22 & M & - & $136 / 85$ & 4.25 & 39.30 & 11.00 & - & - \\
\hline IV-1 & 14 & $\mathrm{~F}$ & - & $127 / 83$ & 5.08 & - & - & - & - \\
\hline IV-2 & 11 & $\mathrm{~F}$ & - & $100 / 70$ & 4.67 & - & - & - & - \\
\hline IV-4 & 3 & M & - & $85 / 50$ & 4.14 & - & - & - & - \\
\hline
\end{tabular}

F, female; M, male; BP, blood pressure; $\mathrm{K}^{+}$, potassium level; PRC, plasma renin concentration (reference range $4.4-46.1 \mu \mathrm{IU} / \mathrm{mL}$ ); PAC, plasma aldosterone concentration (reference range 3.0-35.3 ng/dL). ${ }^{1}$ Subjects carried an identified heterozygous mutation in SCNN1B. ${ }^{2}$ Tested after keeping the subject in a standing position for $2 \mathrm{~h}$.

of hypertension for the purpose of exploring the causes of his hypertension with hypokalemia and provding effective treatment. Renal and adrenal computed tomography scans were normal. Echocardiography showed enlargement of the left atrial wall and increased thickness of the left ventricular posterior wall $(16 \mathrm{~mm})$ and interventricular septum $(14 \mathrm{~mm})$. Biochemical data showed severe hypokalemia (2.74 mmol/L), increased PRC $(69.3 \mu \mathrm{IU} / \mathrm{mL}$; reference range $4.4-46.1 \mu \mathrm{IU} / \mathrm{mL})$, and suppression of PAC $(1.5 \mathrm{ng} / \mathrm{dL}$; reference range $3.0-35.3 \mathrm{ng} / \mathrm{dL}$ ).

Additionally, the other 6 family members presented with early-onset hypertension (Table 1). The proband's father (II-7) was diagnosed with hypertension (180/120 mm Hg) at the age of 20 years, but he did not receive formal treatment as he was without obvious symptoms. His mother was normotensive and in health. The proband's uncle (II-5) presented a high blood pressure $(220 / 120 \mathrm{~mm} \mathrm{Hg})$ at 15 years of age. His blood pressure was poorly controlled after taking inulin and amlodipine. The proband's aunt (II-3) had increased blood pressure $(200 / 150 \mathrm{~mm} \mathrm{Hg})$ at 20 years of age with headache and dizziness. The individual III-5 was diagnosed with hypertension at 20 years of age, and his maximum blood pressure was 190/130 mm Hg. He had severe headache, dizziness, and hemorrhinia. Taking antihypertensive drugs did not resolve hypertension and his blood pressure was still 170/110 mm Hg. During this family screening, it was found that an 8-year-old child (IV-3) had elevated blood pressure $(150 / 100 \mathrm{~mm} \mathrm{Hg})$ without any symptoms. The proband's grandmother (I-2) died of stroke for uncontrolled hypertension (200/?) 3 years ago. Biochemical examination showed hypokalemia in individuals II-3, II-5, II-7, III-5, and III-8. Individuals II-3, II-5, II-7, III-5, III-8, and IV-3 presented with suppressed PAC, and a lower PRC was observed in II-5, III-5, and IV-3 (Table 1).

\section{Genetic Analysis}

A novel and rare heterozygous variant (c.1849C $>A$ ) was identified in $S C N N 1 B$ in the proband. This variant (p.Pro617Thr) altered an amino acid in the PY motif, resulting in thre- 


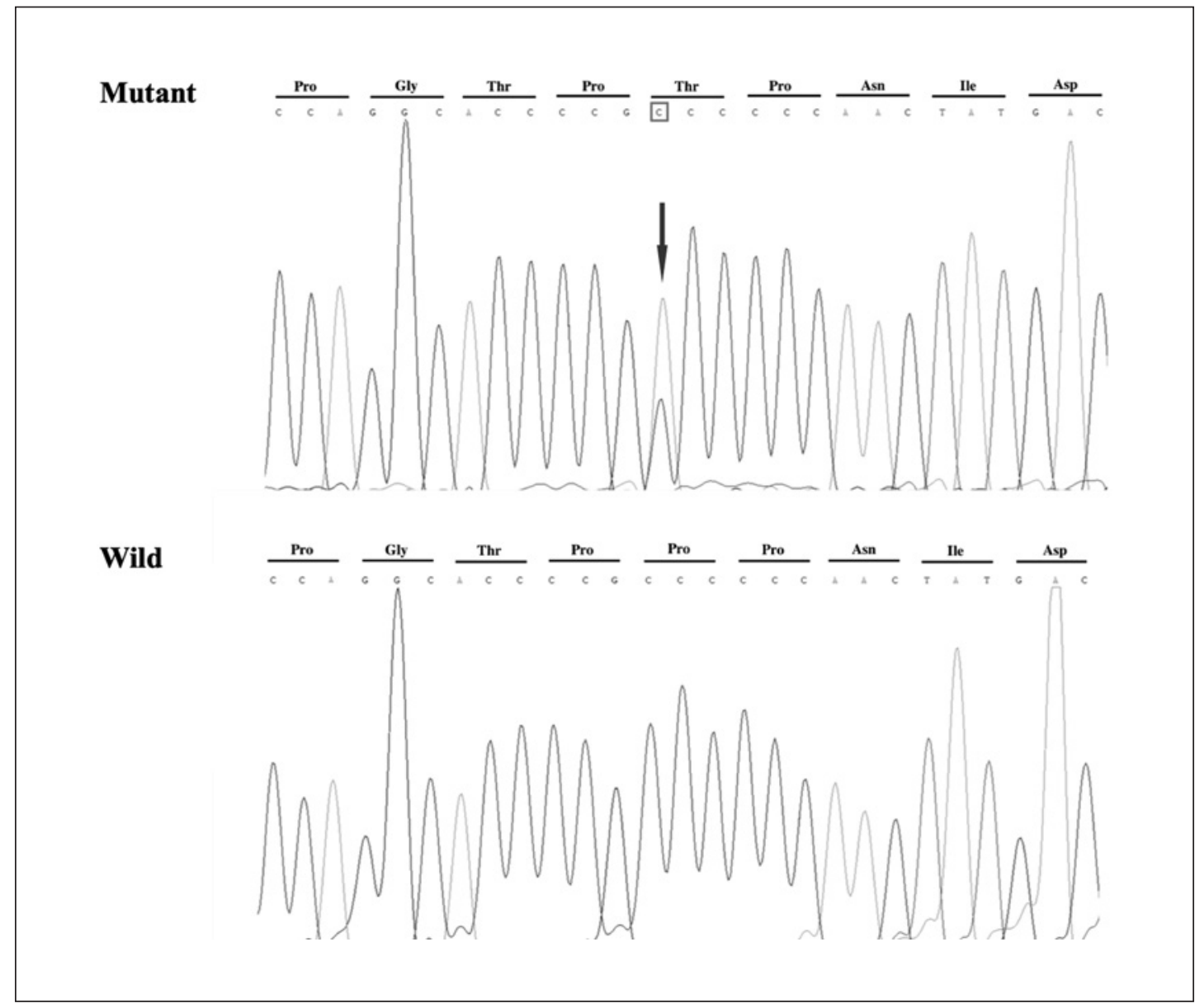

Fig. 2. Genetic sequencing indicates a novel heterozygous mutation (c.1849C>A, p.Pro617Thr) in SCNN1B.

onine instead of proline at codon 617 (Fig. 2). This variant was also detected in individuals II-3, II-5, II-7, III-5, and IV-3. Although the DNA sample of the proband's grandmother was not available, we speculated that this variant was transmitted through the maternal lineage based on the clinical presentation and distribution of the patients. This variant was not found in other family members, 100 hypertensives, or 100 healthy controls. Several databases, including the Human Gene Mutation Database (http://www.hgmd.org), the 1000 Genomes Project database (http://browser.1000genomes.org), and the Exome Aggregation Consortium (http://exac.broadinstitute.org/), did not report this variant. According to the ACMG guideline [12], this missense mutation was considered likely pathogenic. No other SCNN1A, SCNN1B, or SCNN1G variants were detected.

\section{Tailored Medicine for Mutation Carriers}

In this family, all patients carrying the identified $S C N N 1 B$ mutation with hypertension were diagnosed with LS. The proband and his 5 relatives harboring the identified mutation were advised to consume a low-salt diet, to quit alcohol, and to perform a moderate amount of exercise. They were treated with amiloride at 5.0 and $2.5 \mathrm{mg} /$ day for adults and children, respectively. After 1 month of treatment with amiloride, blood pressure and electrolytes were reexamined in each LS patient. Both blood pressure and serum potassium levels returned to the normal range (Table 1). 


\section{Kidney \\ Blood Pressure \\ Research}

\begin{tabular}{l|l}
\hline Kidney Blood Press Res 2020:45:603-611 \\
\hline DOI: 10.1159/000507580 & $\begin{array}{l}\text { @ 2020 The Author(s). Published by S. Karger AG, Basel } \\
\text { www.karger.com/kbr }\end{array}$ \\
\hline
\end{tabular}

Fan et al.: LS Caused by a Novel SCNN1B Mutation

\section{Discussion}

Here, we describe the identification of a novel missense mutation (c.1849C $>$ A) in $S C N N 1 B$ in a Chinese family. Genetic analysis of this site suggested that it was pathogenic through change of the PY motif of $\beta$-ENaC. Genotype-phenotype correlation analysis showed that LS patients carried this mutation and presented heterogeneous phenotypes in this family.

The pathogenesis of LS mainly correlates with the accumulation and activation of ENaC in the distal nephron apical membrane [13]. ENaC consists of 3 homologous subunits, and every subunit has a highly conserved proline-rich sequence called the PY motif, located at the C-terminus $[14,15]$. In this study, we identified a novel mutation (c.1849C >A, p.Pro617Thr), resulting in the alteration of the PY motif of $\beta$-ENaC. To date, 4 SCNN1B mutations have been identified at codon 617 , each of which leads to the replacement of proline with another amino acid, altering the PY motif of $\beta$-ENaC [16-19]. Previous studies have confirmed the pathogenicity of changes at this residue and the crucial role of Pro617 in the regulation of $\beta$-ENaC. Ubiquitin ligase Nedd4 (neural precursor cell expressed, developmentally downregulated 4) binds the PY motif and activates the processes of ENaC ubiquitylation and endocytosis [10, 20], inhibiting the inactivation of ENaC at the cell surface [21]. Overactivation of sodium channels leads to increased sodium reabsorption, and blood volume expansion, resulting in hypertension and potassium loss. Our study has expanded the genetic spectrum of SCNN1B in LS.

The typical clinical features of LS are resistant and early-onset hypertension, hypokalemia, a low renin concentration, hypoaldosteronism, and metabolic alkalosis [22]. Hypertension is the most common symptom in patients with LS. In the family examined in this study, all of the patients presented poorly, with early-onset hypertension. The average age of hypertension onset was 17.5 years in pedigree patients, which is consistent with the characteristics of LS under the age of 30 years [5]. Previous studies have shown that all patients harboring a mutation at codon 617 show early-onset hypertension and that the severity of hypertension is variable, ranging from mild to severe [9]. Target organ damages arise as a consequence of resistant hypertension, including stroke, left ventricular hypertrophy, chronic kidney disease, retinopathy, and even sudden death [23].

Phenotypic variation in LS symptoms was observed based on blood pressure and biochemical examinations [24]. A systematic review revealed hypertension in 92.4\%, hypokalemia in $71.8 \%$, and low plasma aldosterone in $58.2 \%$ of LS patients [9]. In our study, all LS patients had hypokalemia and hypoaldosteronism, but renin levels varied greatly. These results are consistent with those of a previous study showing that a low renin concentration is not a necessary presentation in LS patients [25]. Carriers of the Pro617Leu mutation presented normal plasma potassium concentrations and suppression of both renin and aldosterone in a family [26]. In another family with the same mutation (Pro617Leu), LS patients manifested hypokalemia [27]. Phenotypic heterogeneity was showed between different families and between members of the same family [28]. Genetic and environmental factors may account for phenotypic variation [29], including a dietary habit of low salt intake and other genes controlling sodium reabsorption, which may explain why the proband was hyperreninemic. In addition, variants in 5 other genes can increase the activity of $\mathrm{ENaC}$, resulting in Liddle-like phenotypes [30].

Over the last decade, diagnosis of LS has dramatically improved as a direct result of an increase in our knowledge of the genetic mutations underlying sporadic and familial forms of the disease [31]. LS diagnosis is based on clinical characteristics and genetic evidence. LS should be suspected in patients presenting with early-onset hypertension and hypokalemia, especially associated with a family history of resistant hypertension, stroke, and sudden death. Genetic sequencing is required to confirm the diagnosis after clinical selection. The 
selection of only highly suspected individuals based on typical LS symptoms can lead to patients with LS being overlooked or misdiagnosed due to phenotypic variation. In addition, some complications can be the first symptom noticed in some LS patients. In this study, the proband presented a hemorrhagic stroke. Some patients with severe complications were diagnosed with LS when pathogenic mutations were identified in their first-degree relatives [25]. Excluding other causes leading to hypertension, LS should be considered in all cases of early-onset hypertension, irrespectively of hypokalemia, low renin levels, and family history. Genetic screening should be recommended for first-degree relatives of patients with LS to identify more mutation carriers in the family [32].

Based on the pathophysiology, the specific treatment for LS is ENaC blockers in combination with a low-sodium diet. As a specific $\mathrm{ENaC}$ inhibitor, amiloride is effective in controlling hypertension and correcting the biochemical alterations in LS by binding and inactivating ENaC. Keeping blood pressure within the normal range can assist in avoidance of severe complications [23]. For the proband, controlling hypertension can avoid secondary stroke. Follow-up results show that patients carrying this novel mutation respond exceptionally well to amiloride. Specific therapy was recommended for controlling hypertension and reducing the occurrence of complications in LS.

\section{Conclusion}

We report a novel missense $S C N N 1 B$ mutation in a Chinese family and enrich the spectrum of pathogenic mutations known to cause LS. The disease-causing mutation alters the PY motif by amino acid substitution. This study reminds us that vigilance for premature stroke is always required in LS patients with severe hypertension. Genetic sequencing is recommended for patients suspected to have LS and their first-degree relatives for a timely diagnosis. Accurate diagnosis and early specific treatment can help LS patients avoid target organ damage of hypertension.

\section{Statement of Ethics}

This study was approved by the Ethics Committee of Fuwai Hospital. All of the participants provided written informed consent.

\section{Conflict of Interest Statement}

The authors declare that they have no competing interests.

\section{Funding Sources}

This work was supported by the Non-profit Central Research Institute Fund of Chinese Academy of Medical Sciences (2019XK320057 and 2019XK320058), the National Key Research and Development Program of China (2016YFC1300100), the CAMS Innovation Fund for Medical Sciences (2016-I2M-1-002), the National Natural Science Foundation of China (81600305 and 81974042). 


\section{Kidney \\ Blood Pressure Research}

\begin{tabular}{l|l}
\hline Kidney Blood Press Res 2020;45:603-611 \\
\hline DOI: 10.1159/000507580 & $\begin{array}{l}\text { @ 2020 The Author(s). Published by S. Karger AG, Basel } \\
\text { www.karger.com/kbr }\end{array}$ \\
\hline
\end{tabular}

Fan et al.: LS Caused by a Novel SCNN1B Mutation

\section{Author Contributions}

P.F., Y.-X.L. and X.-L.Z. designed and supervised the research. P.F., D.Z., X.-C.P., K.-Q.Y., Q.-Y.Z., X.-Y.L., W.-J.M., H.-M.Z., L.S., and J.C. collected samples and clinical information. P.F., D.Z., and Y.-T.L. performed the experiments. P.F., D.Z. and Y.Z. performed the data analysis. P.F. and D.Z. wrote this paper. P.F., Y.-X.L. and X.-L.Z. modified this paper. All of the authors reviewed this work.

\section{References}

1 Kearney PM, Whelton M, Reynolds K, Muntner P, Whelton PK, He J. Global burden of hypertension: analysis of worldwide data. Lancet. 2005 Jan;365(9455):217-23.

2 Olsen MH, Angell SY, Asma S, Boutouyrie P, Burger D, Chirinos JA, et al. A call to action and a lifecourse strategy to address the global burden of raised blood pressure on current and future generations: the Lancet Commission on hypertension. Lancet. 2016 Nov;388(10060):2665-712.

3 Burrello J, Monticone S, Buffolo F, Tetti M, Veglio F, Williams TA, et al. Is There a Role for Genomics in the Management of Hypertension? Int J Mol Sci. 2017 May;18(6):18.

4 Simonetti GD, Mohaupt MG, Bianchetti MG. Monogenic forms of hypertension. Eur J Pediatr. 2012 Oct; 171(10): 1433-9.

5 Liu K, Qin F, Sun X, Zhang Y, Wang J, Wu Y, et al. Analysis of the genes involved in Mendelian forms of low-renin hypertension in Chinese early-onset hypertensive patients. J Hypertens. 2018 Mar;36(3):502-9.

6 Wang LP, Yang KQ, Jiang XJ, Wu HY, Zhang HM, Zou YB, et al. Prevalence of Liddle Syndrome Among Young Hypertension Patients of Undetermined Cause in a Chinese Population. J Clin Hypertens (Greenwich). 2015 Nov;17(11):902-7.

7 Liddle GW. A familial renal disorder stimulating primary aldosteronism but with negligible aldosterone secretion. Trans Assoc Am Physicians. 1963;76:199-213.

8 Botero-Velez M, Curtis JJ, Warnock DG. Brief report: liddle's syndrome revisited-a disorder of sodium reabsorption in the distal tubule. N Engl J Med. 1994 Jan;330(3):178-81.

9 Tetti M, Monticone S, Burrello J, Matarazzo P, Veglio F, Pasini B, et al. Liddle Syndrome: Review of the Literature and Description of a New Case. Int J Mol Sci. 2018 Mar;19(3):19.

10 Hanukoglu I, Hanukoglu A. Epithelial sodium channel (ENaC) family: Phylogeny, structure-function, tissue distribution, and associated inherited diseases. Gene. 2016 Apr;579(2):95-132.

11 Yang KQ, Lu CX, Xiao Y, Liu YX, Jiang XJ, Zhang X, et al. A novel frameshift mutation of epithelial sodium channel $\beta$-subunit leads to Liddle syndrome in an isolated case. Clin Endocrinol (Oxf). 2015 Apr;82(4):611-4.

12 Richards S, Aziz N, Bale S, Bick D, Das S, Gastier-Foster J, et al.; ACMG Laboratory Quality Assurance Committee. Standards and guidelines for the interpretation of sequence variants: a joint consensus recommendation of the American College of Medical Genetics and Genomics and the Association for Molecular Pathology. Genet Med. 2015 May;17(5):405-24.

13 Schild L, Canessa CM, Shimkets RA, Gautschi I, Lifton RP, Rossier BC. A mutation in the epithelial sodium channel causing Liddle disease increases channel activity in the Xenopus laevis oocyte expression system. Proc Natl Acad Sci USA. 1995 Jun; 92(12):5699-703.

14 Canessa CM, Schild L, Buell G, Thorens B, Gautschi I, Horisberger JD, et al. Amiloride-sensitive epithelial Na+ channel is made of three homologous subunits. Nature. 1994 Feb;367(6462):463-7.

15 Li YL, Zhang D, Tu H, Muelleman RL. Altered ENaC is Associated With Aortic Baroreceptor Dysfunction in Chronic Heart Failure. Am J Hypertens. 2016 May;29(5):582-9.

16 Rossi E, Farnetti E, Debonneville A, Nicoli D, Grasselli C, Regolisti G, et al. Liddle's syndrome caused by a novel missense mutation (P617L) of the epithelial sodium channel beta subunit. J Hypertens. 2008 May;26(5): 921-7.

17 Sawathiparnich P, Sumboonnanonda A, Weerakulwattana P, Limwongse C. A novel mutation in the betasubunit of the epithelial sodium channel gene (SCNN1B) in a Thai family with Liddle's syndrome. J Pediatr Endocrinol Metab. 2009 Jan;22(1):85-9.

18 Cui Y, Tong A, Jiang J, Wang F, Li C. Liddle syndrome: clinical and genetic profiles. J Clin Hypertens (Greenwich). 2017 May; 19(5):524-9.

19 Inoue J, Iwaoka T, Tokunaga H, Takamune K, Naomi S, Araki M, et al. A family with Liddle's syndrome caused by a new missense mutation in the beta subunit of the epithelial sodium channel.J Clin Endocrinol Metab. 1998 Jun;83(6):2210-3.

20 Schild L, Lu Y, Gautschi I, Schneeberger E, Lifton RP, Rossier BC. Identification of a PY motif in the epithelial Na channel subunits as a target sequence for mutations causing channel activation found in Liddle syndrome. EMBO J. 1996 May;15(10):2381-7.

21 Ronzaud C, Staub O.Ubiquitylation and control of renal Na+ balance and blood pressure. Physiology (Bethesda). 2014 Jan;29(1):16-26. 
22 Warnock DG. Liddle syndrome: an autosomal dominant form of human hypertension. Kidney Int. 1998 Jan; 53(1):18-24.

23 Braam B, Taler SJ, Rahman M, Fillaus JA, Greco BA, Forman JP, et al. Recognition and Management of Resistant Hypertension. Clin J Am Soc Nephrol. 2017 Mar;12(3):524-35.

24 Gong L, Chen J, Shao L, Song W, Hui R, Wang Y. Phenotype-genotype analysis in two Chinese families with Liddle syndrome. Mol Biol Rep. 2014 Mar;41(3):1569-75.

25 Fan P, Lu CX, Zhang D, Yang KQ, Lu PP, Zhang Y, et al. Liddle syndrome misdiagnosed as primary aldosteronism resulting from a novel frameshift mutation of SCNN1B. Endocr Connect. 2018 Dec;7(12):1528-34.

26 Rossi E, Farnetti E, Nicoli D, Sazzini M, Perazzoli F, Regolisti G, et al. A clinical phenotype mimicking essential hypertension in a newly discovered family with Liddle's syndrome. Am J Hypertens. 2011 Aug;24(8):930-5.

27 Caretto A, Primerano L, Novara F, Zuffardi O, Genovese S, Rondinelli M. A therapeutic challenge: Liddle's syndrome managed with amiloride during pregnancy. Case Rep Obstet Gynecol. 2014;2014:156250.

28 Fan P, Zhao YM, Zhang D, Liao Y, Yang KQ, Tian T, et al. A Novel Frameshift Mutation of SCNN1G Causing Liddle Syndrome with Normokalemia. Am J Hypertens. 2019 Jul;32(8):752-8.

29 Bogdanović R, Kuburović V, Stajić N, Mughal SS, Hilger A, Ninić S, et al. Liddle syndrome in a Serbian family and literature review of underlying mutations. Eur J Pediatr. 2012 Mar;171(3):471-8.

30 Jin HS, Hong KW, Lim JE, Hwang SY, Lee SH, Shin C, et al. Genetic variations in the sodium balance-regulating genes ENaC, NEDD4L, NDFIP2 and USP2 influence blood pressure and hypertension. Kidney Blood Press Res. 2010;33(1):15-23.

31 Ahn SY, Gupta C. Genetic programming of hypertension. Front Pediatr. 2018 Jan;5:285.

32 Liu W, Yin X. The research progress of monogenic inherited hypertension. London: IntechOpen; 2019. 